(e-migrinter

\title{
e-Migrinter
}

$18 \mid 2019$

L'ethnographie en migration(s)

\section{Ethnographie et désidentifications}

Deux enquêtes sur les migrations en pentecôtisme (Paris, Istanbul)

\section{Armand Aupiais}

\section{(2) OpenEdition}

\section{Journals}

Édition électronique

URL : https://journals.openedition.org/e-migrinter/1758

DOI : 10.4000/e-migrinter. 1758

ISSN : 1961-9685

Éditeur

UMR 7301 - Migrinter

\section{Référence électronique}

Armand Aupiais, "Ethnographie et désidentifications », e-Migrinter [En ligne], 18 | 2019, mis en ligne le 12 septembre 2019, consulté le 20 mai 2021. URL : http://journals.openedition.org/e-migrinter/1758 : DOI : https://doi.org/10.4000/e-migrinter.1758

Ce document a été généré automatiquement le 20 mai 2021.

Tous droits réservés 


\title{
Ethnographie et désidentifications
}

Deux enquêtes sur les migrations en pentecôtisme (Paris, Istanbul)

\author{
Armand Aupiais
}

\section{NOTE DE L'AUTEUR}

Nous tenons à remercier tous les acteurs (ici anonymisés) de ces deux enquêtes, Amandine Desille, Mélanie Duclos et les relecteurs anonymes de $e$-Migrinter pour leurs corrections et suggestions, et surtout Lydia Zeghmar pour ses relectures critiques extrêmement enrichissantes.

\section{Introduction}

1 Un corpus de recherches constitué dès 1940 en Amérique du Nord (Hunt, 2002) et depuis la fin des années 1960 en Amérique du Sud (Mariano, 2001) explique l'affinité entre la migration rurale-urbaine et l'adhésion au pentecôtisme - un mouvement chrétien associant l'expérience de la renaissance en Christ et l'expression rituelle de dons du Saint-Esprit. Cette adhésion est parfois décrite comme spontanée et émotionnelle chez les populations déracinées ${ }^{1}$, voire associée à une "régression de la religion vers ses formes les plus élémentaires» $(\mathrm{Fer}, 2005)^{2}$. Les enquêtes que nous présentons ici portent sur des aires géographiques où les liens fonctionnels entre pentecôtismes et migrations internationales, sont établis : en Europe de l'Ouest, en situation postcoloniale et diasporique (Demart, 2017) ; et au Moyen-Orient, en situation de transit et d'impasse migratoire (Picard, 2013). Nous interrogeons les migrations en pentecôtisme, soit la construction d'une relation au fait migratoire à partir des espaces religieux, plutôt que le "pentecôtisme en migration », qui supposerait la prééminence de la situation migratoire sur la pratique religieuse. Nos interactions avec les membres de deux Églises transnationales ${ }^{3}$, l'Église Universelle (originaire du Brésil) en France, et l'Église que nous appellerons $\mathrm{La}$ Cascade (originaire des États-Unis) en Turquie ${ }^{4}$ constituent le matériau empirique retenu. 
2 L'ethnographie, qui passe par l'enregistrement, l'ordonnancement et la restitution de réalités socialisés dans des regroupements humains, semble indissociable du partage, si ce n'est entre colons et indigènes, a minima entre un " nous » et un " eux » (Lenclud, 1996). Selon une image classique, si l'ethnographe participe de l'univers de pratiques et de représentations qu'il étudie, il devrait procéder par «distanciation » par rapport à ce «nous»; et s'il y est étranger, il devrait avancer par "dépaysement» et par « approximation » (Beaud \& Weber, 2003 [1997]) du « eux » enquêté. L'emploi de termes relevant du champ lexical de l'enquête, plus qu'il ne trahit la connivence entre pratiques ethnographiques et policières ${ }^{5}$, signale leur affinité plus générale avec l'identification sociographique des populations et de leurs territoires, préalable à toute démonstration. Or là où la police procède par association entre «identifiant territorial» et "qualité pénale» (Jobard, 2006), l'ethnographe balance entre la contrainte d'identifier des catégories statistiques et le devoir de documenter l'existence réelle, localisée et dynamique, de personnes dont les activités débordent ces catégories.

La conversion religieuse et la migration, actions non nécessairement confondues avec les identités confessionnelles et/ou ethniques qui tendent à s'y agréger, sont prises ici comme des "désidentifications ». La notion de désidentification est empruntée à la philosophie politique, où elle peut désigner l'«arrachement à la naturalité d'une place » (Rancière, 1995 : 60) et correspondre à un investissement collectif de l'ordre social, ou plus subjectivement un « écart à soi » faisant advenir un être " pour autre que ce qu'il est, (...) dans un mouvement qui a singulièrement à voir avec qui il est » (Fjeld \& Tassin, 2015). Nous la définissons comme une pratique par laquelle un sujet déjoue certaines assignations. L'ethnographie des migrations thématiserait une sortie de soimême par le départ du sujet et/ou son installation hors des espaces d'assignation ${ }^{6}$, et l'ethnographie des conversions la distance mise par le sujet entre soi et le "monde ", à travers sa participation à l'« œuvre » de Dieu. Cette perspective peut être élargie à de nombreux objets de recherche, et l'ethnographie gagnerait souvent selon nous à être envisagée comme l'observation des dynamiques de désidentification, soit le départ ou l'écart des sujets observés par rapport à des positions sociales hiérarchisées et situées, positions par ailleurs établies dans la dynamique d'enquête. Transférer cette notion en socio-anthropologie ${ }^{7}$ lui impose un relativisme et un perspectivisme méthodologiques. Nous ne préjugeons donc pas l'aspect politiquement émancipateur des désidentifications religieuse et/ou migratoire que nous observons - qui peuvent se traduire par des (ré)assujettissements institutionnels relativement conformes à l'ordre social. Et soulignons que la conversion et l'émigration-installation sont généralement vécues par les acteurs pentecôtistes comme l'exécution d'un plan de Dieu, à travers des récits de conversion et des projets migratoires imbriqués, et profondément optimistes.

Partant d'une discussion sur l'ethnographie des migrations en pentecôtisme, et en passant par la description de relations d'enquête jalonnées d'identifications plus ou moins nettes, nous entendons montrer que l'ethnographie procède toujours par une double désidentification. Désidentification, d'abord, par l'observation et la restitution fidèle des écarts produits par des êtres qui se subjectivent. Désidentification, enfin, par l'adoption du point de vue d'un ethnographe produisant lui-même un écart par rapport à ce qu'il est. Cet article propose donc une amorce de dépassement de la dichotomie héritée du colonialisme entre anthropologie "chez soi » et anthropologie "chez l'autre » (Gullestad et al., 2009). Avec la désidentification, nous proposons un principe unifié de l'ethnographie, sans rejeter la paire conceptuelle distanciation/dépaysement. 
Mesurant ces écarts, l'ethnographie permet en effet d'établir les coordonnées sociales initiales des protagonistes de l'enquête, et leurs positions parfois antagonistes dans les rapports sociaux concrets, y compris (post)coloniaux.

5 Après avoir posé dans la première section le problème de la croyance réelle ou supposée des ethnographes du pentecôtisme, comme mode d'accès (ou obstacle) à la compréhension du religieux, nous nous dégagerons de ce problème pour insister sur l'imbrication de la croyance dans des rapports sociaux produits, actualisés ou négociés dans l'enquête. Dans les deuxième et troisième sections, nous reviendrons donc sur les actions et les identifications dont nous avons été l'objet pour chaque monographie, au regard du contexte migratoire et organisationnel des Églises et des sociétés d'implantation.

\section{Ethnographier le pentecôtisme}

Contrairement aux ethnographes des religions initiatiques, qui peuvent s'approcher par paliers pour découvrir les secrets des systèmes mythico-rituels appréhendés, les ethnographes du christianisme évangélique, religion révélée et prosélyte, feraient face à une "alternative tranchée » entre le choix de leur conversion, ou leur diabolisation (Mary, 2000). Là où l'implication des premiers est relativement tolérée par leurs collègues, la participation des seconds aux activités cultuelles suscite parfois la méfiance des milieux académiques (Mahieddin, 2015 : 462). Ainsi les ethnographes du pentecôtisme mettent au cœur de leur questionnement leur degré de participation aux pratiques, voire d'adhésion aux croyances religieuses. Dire ouvertement son athéisme (Blanes 2006) ou mentir en se présentant comme convertie tout en s'entraînant méthodiquement à la prière (Mézié, 2010); extérioriser et partager ses émotions pendant le rituel (Mossière, 2007) ou refuser de fermer les yeux et de s'agenouiller avec les autres (Mottier 2014) ${ }^{8}$; profiter d'une affiliation passée au protestantisme évangélique (Soothill, 2007) ou au catholicisme charismatique (Aubourg 2011) pour se faire accepter, ou s'appuyer sur une identité protestante mainline pour «ne pas jouer » au pentecôtiste (Lalive d'Épinay, 2007) : autant de choix de postures pour l'ethnographe qui apparaissent comme déterminants dans ce milieu.

7 Le «ludisme méthodologique» (Droogers \& Knibbe, 2011), basé sur le jeu de l'ethnographe et centré sur les modes d'engagement émotionnel du croyant et son «type de jeu " semble heuristique, les manifestations de l'émotion religieuse étant au cœur du discours pentecôtiste (Corten, 1995). Mais le « théâtre vécu » (Leiris, 1996) par les participants à une transe pour obtenir la guérison ou la bénédiction ne saurait être confondu avec le jeu de l'ethnographe qui «se fait croire à lui-même qu'il partage "en un certain sens" les croyances des autres " (Olivier de Sardan, 2000). Dans le cas des Églises où nous avons enquêté, la participation active des fidèles à une "délivrance " ciblée des démons (Universelle) ou à une " percée " généralisée de l'Esprit Saint (Cascade) engage certes une capacité de jeu, mais aussi la conviction que ces entités agissent réellement, suivant des modalités relativement indépendantes de leur mise en scène.

Ces options méthodologiques ne devraient pas occulter un enchevêtrement de rapports sociaux ${ }^{9}$ fondamentaux dans la gestion des frontières entre enquêteurs et enquêtés. Il importe à l'ethnographe soucieux de comprendre les discours et représentations des acteurs de son terrain (point de vue émique) en regard de la domination dans une société (et du point de vue hégémonique qui en découle), de se situer dans les rapports 
sociaux asymétriques et inégalitaires qui structurent cette société et ses relations dans les espaces religieux. Les travaux récents des anthropologues Pamela Millet-Mouity et Sarah Demart illustrent ce caractère déterminant dans le cadrage de l'enquête. La première, ancienne pentecôtiste poussée à la «reconversion » sur son terrain, fût demandée en mariage dans un milieu considérant que, «en tant que jeune femme catégorisée comme noire, [elle avait] forcément fait l'expérience de la conversion » (Millet-Mouity, 2017 : 18). La seconde fût exposée, en vertu de sa "position de femme blanche " et bien que considérée comme convertie, à des avances extra-conjugales rompant avec la morale chrétienne (Demart, 2017: 40). Dans ces deux récits, l'identification morale et religieuse de la femme à marier, comme de l'amante potentielle, est explicitement solidaire de rapports de race et de sexe imbriqués.

9 En quoi nos interactions d'enquête, décisives dans la production du savoir anthropologique (Girola, 1996) permettent-elles de dégager les spécificités inhérentes des liens entre migrations et pentecôtismes? Et comment les contradictions indépassables entre extériorité relative et implication marginale dans des communautés pentecôtistes se sont-elles exprimées dans deux contextes différents, fournissant à ce titre des clés de compréhension sur ces contextes (Althabe \& Hernandez, 2004)? Le travail religieux - ensemble des tâches qui, utiles au fonctionnement des lieux de culte et des entreprises missionnaires, sont inégalement réparties entre les fidèles - nous semble être un point d'observation privilégié pour étudier ces rapports. Nous nous appuierons ici sur les données de nos enquêtes auprès des membres actifs des groupes de jeunesse de deux Églises où, nous étant présenté comme étudiant-chercheur en sciences sociales travaillant sur l'évangélisme, nous participions aux activités cultuelles et missionnaires en veillant à ne pas être directement impliqué comme travailleur religieux.

\section{À Paris : des autochtonies ${ }^{10}$ en tension}

En Europe de l'Ouest, l'adhésion au pentecôtisme comme construction de groupes solidaires dans l'exil (Maskens, 2013) est un fait établi, tout comme sa capacité subjectivante à engendrer des "hétérotopies" en diaspora (Demart, 2017). Un large corpus de recherches aborde, à partir d'études de cas au sein des immigrations africaines en particulier, le rôle de l'implantation des Églises « recrées» (Levitt, 2003) comme " communautés retrouvées » (Fancello \& Mary, 2010), éléments fondateurs des premières formations diasporiques postcoloniales (Harris, 2006) ${ }^{11}$, ou d'identifications originales au «christianisme global» (Haar, 1998). Alors que la rupture généalogique engagée par les pentecôtistes avait été classiquement établie par les sciences sociales en milieu rural, au Brésil (Novaes, 1985), la présence de descendants d'immigrés dans les Églises pentecôtistes «en migration » (Fancello, 2007) d'Europe de l'Ouest reste peu étudiée. Notre enquête à Paris s'intéressait plus particulièrement au travail religieux (quasi-exclusivement non rémunéré) effectué par des descendants d'immigrés africains et afro-caribéens, qui participaient tout particulièrement à l'implantation et à l'indigénisation ${ }^{12}$ d'une organisation transnationale en Europe, l'Église Universelle du Royaume de Dieu (EURD, ou Universelle).

11 L'Universelle a été fondée à Rio de Janeiro par le Brésilien Edir Macedo en 1977. Au cœur de la vague dite "néo-pentecôtiste", elle promeut une théologie de la "foi rationnelle» encourageant les fidèles au sacrifice moral et financier comme voie 
d'accès, contractuelle, à la «vie en abondance » (Gomes, 2009), sur fond d'organisation centralisée fortement investie sur le marché médiatique et dans le jeu politique. Très présente sur les continents africain, américains et européen, elle s'est implantée en France en 1992. Arrivé fin 2011, nous ne recensions sur notre carnet de terrain ni les " origines" supposées, ni les "couleurs" perçues dès lors qu'elles n'étaient pas exprimées par les acteurs eux-mêmes ${ }^{13}$. Si les autochtones subissaient l'« attribution généralisée à l'altérité » (Maskens, 2013 : 154) réservée aux immigrés primo-arrivants ou installés de longue date, la catégorie de "génération de l'immigration" nous semblait trompeuse, l'acte de migrer des ascendants ne faisant pas en soi " origine " (De Rudder, 1998). Après plusieurs semaines d'imprégnation, l'Église apparaissait comme fréquentée par environ 150 membres. Ces membres étaient principalement des immigrés originaires d'Afrique francophone (RDC en particulier), des DOM, et d'Haïti aux réunions en langue française; des immigrés originaires du Portugal, d'Angola, du Brésil et du Cap-Vert aux réunions en langue portugaise; et des descendants d'immigrés africano-caribéens aux réunions du "groupe jeune » ou Force Jeune France $(\mathrm{FJF})$, où nous avions été intégrés comme membre d'une «tribu $»^{14}$. Dans ce groupe les références à la migration, à l'Afrique ou aux Caraïbes, faisaient l'objet de dérision. Par contraste avec les réunions « ordinaires » en langues française et portugaise, la mise à distance des expériences migratoires directes ou indirectes au sein du groupe jeune, associée à la prégnance du répertoire ethno-national dans le discours des fidèles (Aupiais-L'homme, 2017) mettait au défi l'association théorique entre migration et ethnicisation, dévoilant au contraire les ressorts de l'ethnicisation et de la racisation ${ }^{15}$ des autochtones noirs en France. Les usages symboliques de l'ethnicité ne nous semblaient pas constituer le «ferment de mobilisation » indispensable pour parler de "seconde génération" (Simon, 2000: 24), et les acteurs "mettaient en avant une communauté en Christ sans aucune extension ethnique " (Glick Schiller et al., 2006) défiant leur minorisation par rapport aux «citoyens sans passé migratoire reconnu » (Martiniello \& Simon, 2005).

Homme blanc parisien de milieu aisé, dans un groupe de femmes et d'hommes " noirs » de quartiers et familles populaires, nous fîmes d'abord l'objet d'une certaine indifférence, au sens où pratiquement personne ne venait à notre rencontre. Les adeptes nous identifiaient fréquemment comme Portugais lors de nos premiers contacts. La première question de Jonathan, qui deviendrai notre principal contact, fut: «T'es de quelle origine, Portugais? » (février 2012). Une membre s'était élevée un jour contre le procès d'alter-casting (Lyman \& Douglass, 1973) consistant à nous accoler le rôle ethnique considéré comme conforme à notre " couleur ", et avait redéfini notre identité à partir d'une catégorie raciale jugée plus pertinente : « tout ça parce qu'il est Blanc ». Plus tôt, alors que nous étions en train de balayer avec elle la salle de culte, elle avait lancé à un responsable de notre tribu, surpris de nous y voir : «Lui au moins, il nettoie, pas comme certains hommes!» (février 2013), qualifiant notre repli désormais consommé par rapport au groupe des hommes, comme un écart moralement positif par rapport au type de masculinité qui y prévaudrait. Ce fût en tout cas la seule fois qu'une catégorie de "couleur " était utilisée devant nous, ce qui fait écho à une cécité et un mutisme sur les catégories de «race » en France. Une autre «Blanche » prise comme nous pour une Portugaise, et prétextant qu'il n'y avait " presque aucun Français » à l'église, remplaçait cette équation ethnico-raciale $:$ Blanc = Portugais, liée au contexte conjoncturel ; par une autre : Français =Blanc, plus fidèle au contexte structurel ${ }^{16}$ (février 2012). 
13 L'Universelle fut listée comme «mouvement sectaire » dès son implantation en France dans un rapport parlementaire (Gest \& Guyard, 1995) puis empêchée de s'installer dans l'ancien cinéma La Scala, acquis légalement à Paris (Aubrée, 2003). Cette pénalisation de l'organisation contraste avec l'indifférence réservée par les pouvoirs publics aux pentecôtistes africains - une part importante de sa base sociale - de telle sorte que l'Église se situait pratiquement à l'intersection de deux essentialismes républicains : "entre l'ethnie et la secte» (Luca, 2008b), une situation qui contribuerait probablement à renforcer la méfiance qui y régnait et la réaffirmation d'un refus (Mary, 2002: 467) d'accueillir les observateurs. Nos échecs à obtenir le moindre entretien mettaient en évidence un stricte contrôle hiérarchique de la circulation de la parole, et nous nous repliions sur une « observation participante » alliant mimétisme motivé par la volonté de respecter et perturber le moins possible le culte - et présentation de soi comme étudiant «intéressé ${ }^{17}$. Peut-être notre relation avec ses membres était-elle trop affectée par une organisation proscrivant les liens d'amitié entre les fidèles pour éviter qu'ils ne dégénèrent en méfiance et en jalousie (van de Kamp, 2016 : 32). Peut-être aussi nos options morales étaient-elles trop antagoniques avec les prescriptions de la hiérarchie :

14 Diffusion du témoignage ${ }^{18}$ vidéo d'une femme qui, brouillée avec son père depuis dix ans pour des "histoires d'attouchement", lui a finalement pardonné, sans préciser si elle a obtenu une quelconque justice (repentir ou réparations). Aujourd'hui son père fréquente l'Universelle, conclusion saluée par l'assemblée d'un intense "Amen! ". L'exigence de pardon est sans cesse rappelée, y compris dans les cas de consentement ou de silence des parents sur le viol des enfants. (février 2012)

15 Mais la plupart du temps, nous n'assumions pas ouvertement notre désaccord dans nos interactions avec les membres de l'Église, une «éthique de l'aversion [dislike] » qui s'était pourtant avérée heuristique pour l'enquête d'Ilana van Wyk (2013) à l'Universelle. Dans l'exemple ci-dessus notre malaise tenait probablement autant à la distance vis-àvis de la culture pentecôtiste qu'à un écart entre notre expérience de l'ordre patriarcal, socialement à notre avantage (d'où une capacité à en ignorer les enjeux en contexte "familier»), mais heurtant notre sensibilité de sujet dans le cadre de cette manifestation rituelle. Nous manifestions plus opportunément nos réserves face à l'ordre laïc et républicain, et la conscience commune que les croyances et les expériences pentecôtistes étaient, en France, illégitimes au regard d'une "hiérarchie de crédibilité » (Becker, 2013 [1967]) et d'une "hiérarchie de la perception » (Dijk \& Pels, 1996) injustes, puisque favorables à l'agnosticisme et aux religions établies (le catholicisme apostolique, romain, et non charismatique en particulier). Nos discussions sur « ce qu'on nous apprend à l'école » de la laïcité et de l'ancien clergé (membre de la FJF, janvier 2012) ou sur le droit à «prier dans la rue» que s'arrogent certains catholiques (nous-même, décembre 2012) s'appuyaient sur une socialisation comparable sur le territoire français, affectée par son système scolaire et son espace médiatique.

Intégré en janvier 2012 à un sous-groupe de la FJF et malgré 16 visites en moins de quarante jours, un « actif » nous reprochait déjà notre absentéisme. Nous fûmes pris en charge par plusieurs personnes soucieuses de nos «doutes » et "angoisses ", de nos "objectifs» et de notre "progression », nous indiquant des lectures bibliques, priant pour nous, promettant de nous téléphoner sans nous « harceler ». Dans cet univers de contrôle social, les représentants du groupe déploraient que nous n'amenions pas plus 
d'invités à l'église, ou nous demandaient de justifier une absence par une «mission spécifique » au dehors (mars 2012). Lors d'événements de grande ampleur avec les membres d'autres branches de l'Universelle en région parisienne, il arrivait souvent qu'un inconnu nous demande qui nous avait invité. Notre présence n'allait pas de soi.

Cyrus, devenu «chef de tribu » en mon absence, me demande comment j'ai connu l'Église et ce que je fais comme études. J'évoque mon travail sur l'Universelle. Il m'incite à avoir un "objectif» et le poursuivre, une incitation à m'engager dans l'œeuvre: «il faut semer pour récolter »). Par contraste, il relève mon attitude actuelle : "genre le mec qui est là... " J'ironise : "le mec qui est juste là ». Cyrus rit et dit qu'au moins, je suis " honnête ». Il précise que Dieu ne m'a pas envoyé ici " par hasard " mais pour saisir "l'opportunité de Le servir », et il me met au "défi» d'inviter quelqu'un le samedi suivant. (octobre 2012)

Oscillant entre velléités de contrôle du travail religieux, évangélisation et simples " tests ", l'attitude des " actifs » à notre égard indiquait qu'à vouloir rester à la marge du travail religieux, «sur les bords de la structure» (Turner, 1990 [1969] : 125), nous nous enfermions dans le rôle liminaire et inférieur d'un néophyte, exposé à l'humiliation et aux brimades de ses pairs. Dans une réunion réservée aux hommes de notre «tribu » (novembre 2012), l'ancien chef qualifia les membres trop absents de " tocards », de " couilles-molles ", les enjoignant à se mettre à l'écart des « vaillants " et attendre les instructions, ce que nous fîmes sans hésitation, quittant même la réunion avant la fin, pour réduire notre implication.

19 Le refus formel des observateurs n'est pas toujours reproduit par la hiérarchie informelle : un membre "actif » nous avait promis un entretien, quoiqu'il fût toujours absent ou indisponible aux rendez-vous fixés et reportés quatre fois. L'extrait qui suit met en scène un échange conflictuel dans lequel il est directement rappelé à l'ordre par le premier échelon de la hiérarchie formelle (l'ouvrier Alfredo), et indirectement par son pasteur responsable. Les termes que nous y employions, par réaction à la menace et à la diabolisation (au sens propre), rompent avec la diplomatie et la neutralité adoptées jusqu'ici. Ils signalent une certaine saturation vis-à-vis de l'organisation, tout autant que notre imprégnation par son imaginaire démonologique :

Énième rendez-vous avec Tony, membre actif de la FJF, qui m'accorde finalement un entretien, dans l'église. Je cherche un papier pour prendre des notes, et il s'assure que je n'écris pas sur le journal de l'Église, Le Semeur : "Ça, c'est sacré». Assis sous une caméra de surveillance, nous commençons. Il tourne en dérision mes questions sur sa pratique de la musique, reprend ses réponses à deux fois, refuse de parler de l'Église. Alfredo, devenu ouvrier, nous interrompt. Ils parlent à l'écart et Tony me prévient qu'ils vont partir. Nous reprenons trente secondes, puis ils disparaissent et j'attends une demi-heure en devinant la suite. Revenu, Tony me demande d'effacer l'enregistrement et de lui remettre mes notes. Agacé, je déchire la feuille, la jette à la corbeille, et efface l'enregistrement sous ses yeux. "Déçu par [mon] attitude » il m'explique que "le malin" peut agir à notre insu. Je réplique qu'insinuer que le diable agit à travers moi est « insupportable ». Pasteur Daniel passant par-là, fait allusion à un journaliste qui aurait perdu un procès contre l'Église en Angleterre. J'en appelle sans y croire à l'intérêt d'avoir une visibilité au dehors, et demande en tout cas à ne pas être traité comme un «salaud». Départ cordial mais tendu. (février 2013)

21 Selon Rose-Myrlie Joseph (2013), dans une relation d'enquête l'enquêteur pourrait s'efforcer, faute de "points communs" avec ses enquêtés, de créer avec eux des " points de rencontre ». Le " caractère transnational » de l'Universelle, significatif pour plusieurs membres candidats à la migration vers le Brésil et à l'apprentissage de la 
langue portugaise comme " compétence cosmopolite » (Krause, 2011), a certes favorisé nos échanges autour de ce pays que nous connaissions, et de cette langue que nous parlions, initié des conversations dans lesquelles ces projets migratoires s'exprimaient, et en ce sens constitué un point de rencontre avec nos enquêtés. Mais nous avons échoué à "créer des liens" (Joseph, 2013) et développer avec eux une relation d'enquête ouverte d'affection partagée, voire d'amitié (Duclos, 2014). Il y a une leçon à tirer de cet échec. La relation ethnographique peut prendre place jusqu'à un certain point «chez soi » (Ouattara, 2004), sur le territoire d'où l'anthropologue est autochtone; non pas avec «l'autre [immigré] chez soi » (Fancello, 2008), mais avec des enquêtés qui sont d'autres autochtones; et pas non plus "parmi les siens" (MilletMouity, 2017), puisque des rapports sociaux les opposent. En d'autres termes, non autour d'une autochtonie en commun, mais du partage inégalitaire d'autochtonies mises en tension compte-tenu de la position de l'observateur dans les rapports sociaux en général, et à l'église en particulier. Français privilégié, j' étais dépourvu dans la relation d'enquête du capital d'autochtonie "que procure l'appartenance à des réseaux de relations localisés» (Renahy, 2010), dans des milieux populaires immigrés et/ou "noirs » en l'occurrence. Or la logique de transnationalisation par «en bas " prend appui, par opposition au « cosmopolitisme global » des élites (Capone \& Mary, 2012) sur un " cosmopolitisme vernaculaire » (Bhabha, 2007) qui connecte les acteurs sur la base de leur minorisation dans les sociétés "d'accueil», et sans "dépasser le clivage colonial ». Ni immigrant portugais, ni autochtone "noir", mais majoritaire dans le clivage postcolonial (entre autres), nous incarnions d'autant plus - c'est notre hypothèse - la domination " par le haut » des États qu'appréhendent les fidèles, comme l'illustre la dernière injonction de Jonathan à notre encontre : « Travaille pour glorifier Dieu, pas pour glorifier l'État! » (juin 2013).

\section{À Istanbul : ancrages et incertitudes}

Une littérature récente met en évidence le rôle du pentecôtisme pour les migrants originaires des pays du Sud dans les mondes musulmans comme ressource pour l'ancrage urbain (Picard, 2013), le développement d'un entre-soi protecteur (Kaoues, 2017), le lien avec les convertis autochtones (Boissevain, 2014), voire l'intégration du champ théologique (Bava 2016). À Istanbul selon plusieurs auteures, les Églises agiraient comme "réseau dense et durable de relations » (Danış 2006), « plateforme de rencontres sociales » (Suter, 2012) ou d'alimentation en « énergie spirituelle ( (Akalın, 2016) pour les migrants chrétiens « en transit ». D'autres abordent la conversion depuis l'islam de demandeurs d'asile iraniens et afghans en situation de «transit géographique et spirituel» (Leman, 2007), comme stratégie pour obtenir leur réinstallation en "Occident", ou s'identifier à la «modernité » (Akçapar, 2006) dans le cadre d'une "gouvernance biopolitique" des frontières séparant les désirables des indésirables (Fine, 2013). Tous considèrent donc les populations originaires des Suds dans leur «transit» vers les pays du Nord, et Istanbul comme un espace d'accumulation du capital social nécessaire à la poursuite des carrières migratoires. Suivant notre intérêt pour le travail religieux (toujours massivement non rémunéré), effectué principalement par des immigrantes et immigrants dans l'«impasse» (Aslan \& Pérouse, 2003) et des demandeurs d'asile souffrant d'un gouvernement par l'« incertitude » (Biehl, 2015), nous suivions les trajectoires de fidèles actifs bravant 
l'expérience d'une « vie temporairement en pause, gelée » (ibid : 69) par la propagation $\mathrm{du}$ « feu » de l'Esprit.

L'Église Cascade est dirigée par un acteur majeur du réveil néo-pentecôtiste global (Brown, 2011 : 353-4) initié en Amérique du Nord ${ }^{19}$ dans les années 1990, parfois qualifié de "réveil de l'effusion " en Floride, où cette vague fut particulièrement vive (Hunt 2009). Sa théologie est axée sur des manifestations spectaculaires du «feu » de l'Esprit Saint, comme le « rire sacré » (Kantel, 2006). Officiellement centralisée à Tampa, l'Église Cascade s'apparente à une organisation transnationale négociée relativement flexible et polycentrée (Levitt, 2003). La branche turque, fondée en 1999 par un converti turcoétasunien de famille anciennement musulmane, est émettrice de missionnaires formés à l'Institut Biblique d'Istanbul, qui circulent entre Afrique, Asie, et Europe, tissant un "réseau ouvert» articulé autour de personnalités charismatiques fortes (Oro et al., 2012). Le contact fut facilement établi lors de notre première visite en octobre 2013 : l'épouse du pasteur-fondateur eu même l'air ravie que nous venions « juste observer ». À l'opposé de notre expérience en France, aidé par un pasteur qualifiant systématiquement dans son travail cultuel la nationalité et/ou l'ethnicité des personnes touchées par la grâce et perdant connaissance devant l'assemblée, nous pouvions recenser les origines des fidèles. La Cascade comptait moins de 100 membres réguliers, répartis entre le public des réunions en langue anglaise (originaires d'Afrique, d'Asie, d'Europe), celui des réunions en langue turque (originaires de Turquie, d'Iran et de Syrie notamment), et le groupe jeune ou Dunamis, dont les membres étudiants, migrants internes, internationaux, ou demandeurs d'asile, participaient à toutes les réunions. Concevant a priori les migrants et demandeurs d'asile originaires des Suds dans cette Église comme une population en transit, nous constaterons l'importance de la distinction analytique entre transit et demande d'asile ${ }^{20}$, cette dernière ayant pour premier objectif la légalité de statut, et non une destination concrète (Biner, 2014). Deux demandeurs d'asile irakien et syrien par exemple, entrés au service de l'Église en 2014, ayant intégré et gravi les échelons de la hiérarchie formelle, n'excluaient en 2016 ni de s'installer durablement à Istanbul, ni de poursuivre leur formation théologique à Tampa en Floride. Il faut aussi nuancer l'importance des « réseaux » offerts par l'Église dans la migration, ceux-ci s'épuisant souvent avec l'arrivée en Turquie, où les inégalités de statut légal et de capital culturel priment sur les déterminants économiques (Kalaylıoğlu, 2015). La Cascade, quoique ses représentants la proclament comme une "famille», offre peu d'espace-temps communautaires propices au développement de solidarités réticulaires: le repas partagé y fait même défaut ${ }^{21}$. De plus, les pasteurs tiennent des discours condamnant la migration vers l'Europe de l'Ouest, et attribuent des «dons» et des rôles dans la division du travail missionnaire - une femme nigériane ainsi appelée à sauver des "âmes de femmes musulmanes » en Turquie (juin 2015) - qui incitent à prendre en compte l'Église et les « dons » de l'Esprit Saint comme des institutions médiatrices dans l'élaboration des projets de mobilité ou d'installation.

Nous étions un des rares Européens "mobile » et doté de papiers à l'Église, où nous participions, dans le prolongement de notre socialisation à l'Universelle, aux chants et aux prières, de moins en moins sensible (parce qu'habitué) au "flot de paroles déversées pêle-mêle, accompagnées de larmes, de cris » (Fer, 2005), et ici de fou-rires. Les membres de l'Église nous prenaient souvent pour un Turc, à commencer par le pasteur de l'Église, qui reposa la question de notre origine une seconde fois. Exposé au 
travail missionnaire de l'Église en ville, ressort essentiel de la participation des migrants, nous cédions, par intérêt pour notre enquête, du terrain à l'évangélisme :

J'ai croisé par hasard trois fidèles de Waterfall sur l'avenue İstiklal. Aziz (demandeur d'asile iranien azéri, je ne le connais pas encore) me suit du regard, me talonne, puis m'aborde et sollicite en turc un peu de mon temps. Je reconnais alors Nina (originaire du sud-est de la Turquie, citoyenne étasunienne) et Jamie (migrante philippine) avec lui. Nous parlons un peu, j'explique ma longue absence par mon voyage en France et promets de revenir à l'église. Mais ils veulent me faire "accepter Jésus». Je refuse, dis être mal à l'aise sur cette avenue, avec cette foule. Ils insistent et proposent de se mettre à l'écart du flux des passants. Je cède, ferme les yeux, répète après Nina. Le mercredi suivant Jamie et Aziz sont ravis de me revoir à l'église. (février 2014)

Cette «acceptation" avait peu à voir avec la « combinaison mentale et émotionnelle, qui implique la capitulation d'une forme de vie pour une autre» définie par l'anthropologue Benetta Jules-Rosette (1975) et revendiquée comme mode d'accès privilégié à la compréhension (Jules-Rosette, 1978) dans un mouvement religieux marqué par l'institution des "charismes », ou "dons » de l'Esprit. La pratique des évangélistes ${ }^{22}$ consiste ici à interpeller des passants et leur poser deux questions rituelles : «Vous a-t-on déjà dit que Dieu vous aime et qu'Il a un plan pour votre vie? » et «Si vous mourriez à cet instant, êtes-vous certain d'être sauvé? »; puis à leur proposer de prier pour eux et/ou d'accepter Jésus. Les personnes « sauvées » lors de ces séances sont comptabilisées et rapportées devant le reste de l'assemblée lors du culte dominical ${ }^{23}$. Malgré notre implication, nous ne fûmes jamais considérés comme un membre converti par nos interlocuteurs :

J'accompagne un groupe d'évangélisation composé d'Ismail et trois autres syriennes, à Yeşilköy, au Sud-Ouest d'Istanbul. Dans la voiture, il me demande si je suis " croyant». Je déclare partager à l'église des "émotions " qui me plaisent, et évoque des " origines [background] chrétiennes", déclenchant un discours attendu sur l'importance de renaitre en Christ. Sur place, la responsable du groupe prévient que nous commençons par une "prière en langues ", j'acquiesce en me disant peu "expérimenté» et elle réplique (évidemment) que ce n'est pas une question d'expérience. Nous nous séparons en deux groupes. Finalement, les trois femmes me laissent seul avec Ismail et je commence à croire que c'est moi qu'il évangélise. J'explique ne pas me définir comme "croyant" car fréquentant peu l'église. Nous partageons nos expériences sur le difficile apprentissage de la langue turque. Il se définit comme Assyrien, comme pense-t-il la plupart des habitants de Yeşilköy (les Syriaques d'Istanbul sont la cible de cette séance d'évangélisation), et insiste sur l'atmosphère internationale qui prouve que La Cascade est LA véritable Église, celle où se manifeste la présence de Dieu. Soudain Ismaël s'éloigne vers le rivage. La démarche maladroite, les mains dans les poches, sa silhouette se détache sur le ciel gris flanqué d'un soleil jaune mat caché par les nuages et traversé d'un long trait scintillant à l'horizon. Après trente secondes, je m'approche timidement. Il me raconte avoir vu la mer pour la première fois et avoir rencontré Jésus ici même il y a un an, en compagnie d'une évangéliste étasunienne venue participer au Eurasian Camp Meeting. Il me montre l'endroit exact où il a été "touché". (février 2015)

28 Ismail, sur les bases d'une extranéité en partage auto-évidente dans la conversation difficultés avec la langue, compréhension hasardeuse des groupes ethniques et de leurs ancrages locaux, expérience rituelle cosmopolite dans l'église - donne ici à voir l'image émouvante d'un sujet affecté par sa rencontre personnelle avec Jésus. Du point de vue de l'ethnographe, assumer ses émotions, ses origines culturelles, son expérience 
pratique ou son engagement dans l'Église comme fondement de l'identité et de la pratique religieuses, sont autant de techniques de présentation de soi à la fois inclusives et volontairement auto-excluantes par rapport à l'institution. Nous connaissions en effet l'exigence évangélique de la rencontre personnelle avec le Christ, l'idée que la foi est indépendante d'une "religiosité » systématiquement décriée par les pasteurs, et leur critique des émotions comme moteur de l'action ${ }^{24}$. Mais l'émotion partagée dans la prière pouvait être interprétée comme le gage de nos visites, rejoignant une lecture générale expliquant la présence de l'ethnographe par un appel de Dieu. Cette lecture nous renseigne d'ailleurs sur le prisme missionnaire à travers lequel sont conçues les migrations: un pasteur camerounais ${ }^{25}$ rattaché au réseau Cascade interpréterait notre présence et notre installation en Turquie comme répondant à un appel de Dieu (mai 2016).

La Cascade est listée parmi les "organisations missionnaires" coupables d'une "opération de christianisation de la Turquie " (Bayzan, 2004) dans plusieurs essais anti-missionnaires complotistes, dont un signé par un ancien employé de la direction des renseignements (Yıldırım, 2005). Cette littérature a (re)fleuri en début de siècle, sous les auspices de franges politiquement dominantes dans le pays, accompagnant la perpétration de violences anti-chrétiennes (Cheviron \& Pérouse, 2016:241) qui ont culminé avec l'assassinat de trois protestants évangéliques à Malatya (Anatolie de l'Est) en 2007 (Kutlutürk, 2010). La répression subie à la même époque est parfois évoquée à La Cascade: les membres auraient été à plusieurs reprises empêchés par les forces de police de distribuer des Bibles ou des prospectus, une activité légale bien que souvent entravée ${ }^{26}$. Les missions d'évangélisation auxquelles nous étions invités à participer ${ }^{27}$ nous intéressait particulièrement dans la mesure où elles intervenaient sur des territoires délimités à la rencontre de personnes définies selon leurs origines supposées, et nous renseignaient du même coup sur une certaine cartographie ethnique et migratoire de la ville par les évangéliques. S'impliquer dans ces activités, c'était se risquer à un "jeu de la croyance" (Olivier de Sardan, 1988), parfois heuristique :

30 J'ai suivi une évangélisation à Aksaray avec Jamie, Amid (demandeur d'asile iranien), et Liza (étudiante sud-soudanaise). Dans le centre commercial souterrain, après une joute théologique avec deux syriens (Jamie parle, Liza traduit), sur la nature de Jésus comme "fils» ou simple «messager» de Dieu, Jamie décide d'aborder deux hommes burkinabés, francophones, dont l'un s'éclipse illico. Ce que je craignais arrive : je suis sollicité pour la traduction. (Amid réalise avec joie que notre groupe maîtrise les langues anglaise, arabe, persane, turque et française). Je traduis la première question, l'homme répond amusé que oui, un Témoin de Jéhovah lui a déjà dit que Dieu l'aimait. Je traduis la seconde question et l'homme répond qu'il ira au paradis, n'ayant jamais ni tué, ni volé. Jamie précise à grand renfort de citations de la Bible, que seuls ceux qui connaissent Jésus seront sauvés, et qu'll est mort pour nos péchés. Je maintiens mon rôle d'interprète, bien qu'incapable de traduire les versets cités: ma méconnaissance de la Bible se dévoile. (avril 2015)

31 En endossant le rôle de médiateur "entre des groupes et des codes distincts " en contexte multiethnique, nous faisions l'expérience d'un cosmopolitisme dont le potentiel universaliste n'est "pas distribué de façon égale parmi les habitants de la métropole» (Velho, 2015). Originaire d'Europe de l'ouest, doté de papiers et reconnu comme relativement qualifié sur le marché du travail formel et informel ${ }^{28}$, nous bénéficiions d'un accès facilité par notre apparence extérieure à des espaces difficiles à 
pratiquer pour des personnes perçues comme femmes et/ou étrangères, en particulier. Pourtant, nous partagions avec elles un certain " cosmopolitisme de charisme », limité à la situation rituelle (Mossière, 2014), et un ancrage relativement incertain dans la ville. L'expérience de notre installation se déployait en effet, non dans l'insécurité, mais bien dans l'incertitude quant à la durée du séjour et à la qualité des ressources disponibles ${ }^{29}$ sur place, une incertitude (inégalement) partagée avec certains membres de la Cascade. Incertitude sur nos projets (de circulation, d'installation) à Istanbul et en Turquie. Incertitude sur la fonction de l'Église, ou de l'ethnographie, dans l'élaboration de ces projets. Trois minutes après avoir défini, la Turquie comme sa "terre promise ", dans un entretien-récit d'exil jalonné d'expériences douloureuses, Jamie nous encourageait ainsi à venir au prochain culte : « Je ne sais pas ce que tu y trouveras, mais tu y trouveras quelque chose » (juin 2015).

\section{Conclusion}

32 À Paris comme à Istanbul, le public était morcelé selon une catégorisation ethnonationale lisible dans les discours et les pratiques d'évangélisation. Partant d'une conception de la fabrique identitaire dans l'ordre du projet, plutôt que des origines, et compte-tenu des projets de circulation ou d'installation impulsés par les Églises ellesmêmes, nous pensons qu'il faut nuancer la pertinence de catégories d'analyse telles que les " générations de l'immigration » ou les « migrations de transit ». Les récits de soi et les éthiques mises en œuvre par le pentecôtisme permettent aux sujets d'affronter les contradictions vécues entre autochtonie réelle et assignation systématique à l'altérité pour les descendants d'immigrés en Europe, entre lieu de transit et situation d'impasse migratoire au Moyen-Orient.

Imprégné de l'imaginaire français des «sectes » comme mettant en péril la liberté de penser des individus-citoyens (Luca, 2008a: 70-71) et initié à l'imaginaire turc des «missions» remettant en cause l'intégrité confessionnelle de la Nation (Gözaydın, 2009), nous veillions initialement à nous départir du regard porté par le «normal » sur les «discréditables" (Goffman, 1975), par le «référent" sur les "différents" (Guillaumin, 1979). L'échec et le succès relatifs à créer des liens tiennent certainement au contraste entre l'homogénéité sociale de l'Universelle - Église prolétaire, précaire, noire, avec un groupe de jeunesse autochtone et francophone - et la mixité relative de la Cascade - Église résolument polyglotte réunissant autochtones, migrants et "mobiles» internes et internationaux de divers statuts administratifs et positions économiques. Mais l'ethnographie des migrations en pentecôtisme peut-elle être conçue, aux antipodes de l'enquête policière qui établit les identités, comme une ethnographie de la désidentification? Désidentification par la conversion, entendue comme un effort des sujets pour se projeter dans un « imaginaire communautaire » - la «véritable Église » - et une "utopie collective» (Gallissot, 2004) - le «Royaume de Dieu ». Désidentification caractéristique des situations diasporique et d'extranéité, ou migratoire et de déracinement, enfin (Tassin, 2008).

Ces situations d'enquête "chez soi » et non "parmi les siens» en France, et "chez l'autre " mais parmi d'autres "autres" en Turquie, mettent en lumière le jeu d'oppositions sujet/objet, connaitre/croire, personne/individu, recherche/implication entre enquêtés et enquêteur (Morice 2005), et l'aspect désidentificatoire de l'expérience ethnographique ${ }^{30}$. Impliqué dans les activités du culte, nous étions objectivés par des 
personnes connaissant notre statut social et/ou questionnant notre présence à l'église. Notre statut dans les rapports sociaux : autochtone majoritaire à Paris (dominant dans la division du travail et des ressources autochtones) et immigré du Nord à Istanbul (dominant dans la division internationale du travail et des ressources migratoires), était cependant estompé dans les espaces et les temps rituels. À Paris, d'abord perçu comme immigré par des autochtones, puis redouté comme potentiel agent de l'État, l'échec à créer des points de rencontre sans recourir à des référents transnationaux témoignait de l'ampleur des inégalités entre autochtones, «blancs » et "non-blancs » notamment. À Istanbul, d'abord perçu comme autochtone par des immigrés, puis mobilisé comme évangéliste amateur, notre extranéité rendait possible une rencontre, un partage des émotions (Mossière, 2007) et révélait la capacité des évangéliques à faire de tout étranger un outil de leur travail missionnaire. Plus que l'analogie entre rites de passage et "travail de terrain » (Da Matta, 1984) ou la promotion du "jeu sérieux » comme mode d'accès aux vérités des acteurs (Droogers \& Knibbe, 2011), nous postulons donc la tendance de l'observateur à produire, par la pluralité des identifications dont il fait l'objet dans l'enquête, un "écart » au regard de soi-même et de sa condition. Cet écart permet de mieux mettre en lumière les ambivalences liées à la multiplicité des rapports sociaux mis en jeu par ses enquêtés dans leurs pratiques collectives (Dunezat, 2004 apud Pfefferkorn, 2011) - sans préjuger, une fois encore, des finalités émancipatrices ou aliénantes de cette mise en jeu. L'ethnographie de la conversion et de la migration comme désidentifications, se double ainsi d'une expérience ethnographique par désidentification de l'ethnographe. À charge pour lui de mesurer les implications scientifiques et politiques de cet écart souvent éphémère.

\section{BIBLIOGRAPHIE}

Akalın, Ayse (2016) “Biz Lejyonerleriz !” : İstanbul'daki Filipinli Ev İşçileri, in Bora, E. ; Zorlu, N. ; Ateş, S. (éds.) İstanbul Kimin şehri? Kültür, Tasarım, Seyirlik ve Sermaye, İstanbul, Metis, 408 p.

Akçapar, şebnem Köşer (2006) Conversion as a Migration Strategy in a Transit Country : Iranian Shiites Becoming Christians in Turkey, International Migration Review, vol. 40, n 4, pp. 817-853.

Althabe, Gérard ; Hernandez, Valeria A. (2004) Implication et réflexivité en anthropologie, Journal des anthropologues, $\mathrm{n}^{\circ}$ 98-99, pp. 15-36.

Aslan, Mustafa ; Pérouse, Jean-François (2003) Istanbul : le comptoir, le hub, le sas et l'impasse. Fonctions dans le système migratoire international, Revue européenne des migrations internationales, vol. 19, n 3, pp. 173-204.

Aubourg, Valérie (2011) L'Église à l'épreuve du Pentecôtisme : une expérience religieuse à l'île de la Réunion, Saint Denis, Université de la Réunion, 820 p., Th. Doct. : Anthropologie-Ethnologie, Saint Denis : 2011.

Aubrée, Marion (2003) France, in Corten, A. ; Dozon, J.-P. ; Oro, A. P. (éds.) Les nouveaux conquérants de la foi. L'Église Universelle du Royaume de Dieu (Brésil), Paris, Khartala, pp. 159-163. 
Aupiais-L'homme, Armand (2017) Un pentecôtisme transnational entre migration et autochtonisation : l'Église universelle du royaume de Dieu en région parisienne, in Fer, Y. ; Malogne-Fer, G. (éds.) Le protestantisme à Paris. Diversité et recompositions contemporaines, Genève, Labor et Fides, pp. 257-282.

Bava, Sophie (2016) Migrations africaines et christianismes au Maroc. De la théologie des migrations à la théologie de la pluralité religieuse, Les Cahiers d'Outre-Mer, n² 274, pp. 259-288.

Bayzan, Ali Rıza (2004) Küresel vaftiz : Misyoner örgütlerin Türkiye ve Türk Cumhuriyetlerini Hristiyanlastırma operasyonu, İstanbul, IQ Kültürsanat Yayıncılık, 400 p.

Beaud, Stéphane ; Weber, Florence (2003) Guide de l'enquête de terrain : produire et analyser des données ethnographiques, Paris, La Découverte, 356 p.

Becker, Howard S. (2013) De quel côté sommes-nous ? in Fassin, D. ; Lézé, S. (éds.) La question morale. Une anthologie critique, Paris, Presses universitaires de France, pp. 475-489.

Bhabha, Homi K. (2007) Les lieux de la culture : une théorie postcoloniale, Paris, Payot, 414 p.

Biehl, Kristen Sarah (2015) Governing through Uncertainty : Experiences of Being a Refugee in Turkey as a Country for Temporary Asylum, Social Analysis, vol. 59, $\mathrm{n}^{\circ}$ 1, pp. 57-75.

Biner, Özge (2014) From Transit Country to Host Country : A study of "Transit" Refugee Experience in a Border Satellite City, Van, Eastern Turkey, in Karaçay, A. B. ; Üstübici Ayşen (éds.) Migration to and from Turkey: Changing Patterns and Shifting Policies, Istanbul, Isis, pp. 73-119.

Blanes, Ruy Llera (2006) The atheist anthropologist : believers and non-believers in anthropological fieldwork, Social Anthropology, vol. 14, pp. 223-234.

Boissevain, Katia (2014) Migrer et réveiller les Églises : Diversification des cultes chrétiens en Tunisie, L'Année du Maghreb, n 11, pp. 105-121.

Brown, Candy Gunther (2011) Global Awakenings : Divine Healing Networks and Global Community in North America, Brazil, Mozambique, and Beyond, in Gunther Brown, C. (éd.) Global pentecostal and charismatic healing, New York, Oxford University Press, pp. 351-365.

Capone, Stefania ; Mary, André (2012) Les translogiques d'une globalisation religieuse à l'envers, in Argyriadis, K. ; Capone, S. ; Torre, R. de la ; Mary, A. (éds.) Religions transnationales des Suds Afrique, Europe, Amériques, Louvain-la-Neuve, Academia-l'Harmattan, pp. 49-61.

Cheviron, Nicolas ; Pérouse, Jean-François (2016) Erdoğan nouveau père de la Turquie?, Paris, Éditions François Bourin, 435 p.

Corten, André (1995) Le Pentecôtisme au Brésil : émotion du pauvre et romantisme théologique, Paris, Karthala, 307 p.

Da Matta, Roberto (1984) Relativizando : uma introdução a antropologia social, Rio de Janeiro, Vozes, $246 \mathrm{p}$.

Danış, Didem (2006) “Attendre au Purgatoire” : Les réseaux religieux de migrants chrétiens d'Irak en transit à Istanbul, Revue européenne des migrations internationales, vol. 22, n 3, pp. 109-134.

De Rudder, Véronique (1998) Identité, origine et étiquetage. De l'ethnique au racial, savamment cultivés..., Journal des anthropologues, $\mathrm{n}^{\circ}$ 72-73, pp. 31-47.

Debarbieux, Bernard (2014) Enracinement - Ancrage - Amarrage : raviver les métaphores, L'Espace géographique, vol. 43, $\mathrm{n}^{\circ} 1$, pp. 68-80.

Demart, Sarah (2017) Les territoires de la délivrance. Le réveil congolais en situation postcoloniale (RDC et diaspora), Paris, Karthala, 323 p. 
Dijk, Rijk van ; Pels, Peter (1996) Contested authorities and the politics of perception : deconstructing the study of religion in Africa, in Werbner, R. ; Ranger, T. (éds.) Postcolonial identities in Africa, London, Zed Books, pp. 245-270.

Droogers, André ; Knibbe, Kim (2011) Methodological Ludism and the Study of Religion, Method and Theory in the Study of Religion, $\mathrm{n}^{\circ} 23, \mathrm{pp} .283-303$.

Duclos, Mélanie (2014) Que la relation d'enquête soit aussi d'amitié, Revue ¿ Interrogations ?, $\mathrm{n}^{\circ} 18$ [Disponible sur Internet].

Dunezat, Xavier (2015) L'observation ethnographique en sociologie des rapports sociaux : sexe, race, classe et biais essentialistes, Sociologies (La recherche en actes) [Disponible sur Internet]

Dunezat, Xavier (2004) Chômage et action collective. Luttes dans la lutte. Mouvements de chômeurs et chômeuses de 1997-1998 en Bretagne et rapports sociaux de sexe, Versailles, Université de VersaillesSaint-Quentin-en-Yvelines, 708 p.,

Th.Doct. : Sociologie : Versailles : 2004.

Fancello, Sandra (2008) Travailler sans affinité : l'ethnologue chez les “convertis”, Journal des anthropologues, $\mathrm{n}^{\circ}$ 114-115, pp. 65-90.

Fancello, Sandra (2007) Réveil de l'ethnicité akan et pentecôtisme 'indigène' en Europe, Diversité urbaine, vol. $7, \mathrm{n}^{\circ} 1$, pp. 51-67.

Fancello, Sandra ; Mary, André (2010) Introduction, in Fancello, S. ; Mary, A. (éds.) Chrétiens africains en Europe : prophétismes, pentecôtismes \& politique des nations, Paris, Khartala, pp. 11-40.

Fer, Yannick (2005) Genèse des émotions au sein des Assemblées de Dieu polynésiennes, Archives de sciences sociales des religions, $\mathrm{n}^{\circ} 131-132$, pp. 143-163.

Fine, Shoshanna (2013) The Christianisation of Afghan and Iranian Transit Migrants in Istanbul : Encounters at the Biopolitical Border, Centre on Migration, Policy and Society, Working Paper $n^{\circ} 104$, 14 p. [Disponible sur Internet].

Fjeld, Anders ; Tassin, Étienne (2015) Subjectivation et désidentification politiques. Dialogue à partir d'Arendt et de Rancière, Ciencia Política, vol. 10, n 19, pp. 193-223.

Gallissot, René (2004) Identité / identification, in Mondher, K. ; Rivera, A. ; Gallissot, R. (éds.) L'imbroglio ethnique en quatorze mots clés, Lausanne, Payot, pp. 133-144.

Gest, Alain ; Guyard, Jacques (1995) Rapport fait au nom de la commission d'enquête sur les sectes, Paris, Assemblée Nationale [Disponible sur Internet].

Girola, Claudia (1996) Rencontrer des personnes sans abri. Une anthropologie réflexive, Politix, vol. $9, \mathrm{n}^{\circ} 34$, pp. 87-98.

Glick Schiller, Nina ; Çaglar, Ayşe ; Guldbrandsen, Thaddeus C. (2006) Beyond the ethnic lens : Locality, globality, and born-again incorporation, American Ethnologist, vol. 33, n 4, pp. 612-633. Goffman, Erving (1975) Stigmate : les usages sociaux des handicaps, Paris, Éditions de minuit, 175 p. Gomes, Edlaine (2011) A Era das Catedrais : a autenticidade em exibição, Rio de Janeiro, Garamond, $268 \mathrm{p}$.

Gomes, Edlaine (2009) "Fé racional" e "Abundância" : família e aborto a partir da ótica da Igreja Universal do Reino de Deus, Sexualidad, Salud y Sociedad, n² 2, pp. 97-120.

Gonzalez, Philippe (2014) Être affecté : petite phénoménologie de la « prophétie » charismatique (et de ses conséquences), Sociologies (Dossiers) [Disponible sur Internet]. 
Gözaydın, İşar (2009) Diyanet : Türkiye cumhuriyeti'nde dinin tanzimi, İstanbul, İletişim Yayınları, $352 \mathrm{p}$.

Guillaumin, Colette (1979) Question de différence, Questions Féministes, nº 6, pp. 3-21.

Gullestad, Marianne ; Lien, Marianne Elisabeth ; Melhuus, Marit (2009) Anthropologie « chez soi » et anthropologie « chez l'autre », Ethnologie française, vol. 39, n², pp. 206-215.

Haar, Gerrie ter (1998) Halfway to paradise : African Christians in Europe, Cardiff, Cardiff Academic Press, 220 p.

Harris, Hermione (2006) Yoruba in diaspora : an African church in London, New York, Palgrave Macmillan, 294 p.

Hunt, Stephen (2009) The Florida ‘Outpouring' Revival : A Melting Pot for Contemporary Pentecostal Prophecy and Eschatology?, PentecoStudies : An Interdisciplinary Journal for Research on the Pentecostal and Charismatic Movements, vol. 8, $n^{\circ} 1$, pp. 37-57.

Hunt, Stephen (2002) Deprivation and Western Pentecostalism Revisited : The Case of "Classical" Pentecostalism, PentecoStudies : An Interdisciplinary Journal for Research on the Pentecostal and Charismatic Movements, vol. 1, $\mathrm{n}^{\circ} 1$, pp. 1-32.

Joseph, Rose-Myrlie (2013) Implication dans la recherche : des points communs aux points de rencontre, in Gaulejac, V. de ; Giust-Desprairies, F. ; Massa, A. (éds.) La recherche clinique en sciences sociales, Toulouse, Érès, pp. 133-150.

Kalaylığlu, Yasemin Akis (2015) Uluslararası Göç Bağlamında Bourdieu'nun Kavramları : Ankara'da Yaşayan İranlılar Örneği, İdeal Kent - Kent Araştırmaları, nº 14, pp. 182-205.

Kamp, Linda van de (2016) Violent conversion: Brazilian Pentecostalism and urban women in Mozambique, Woodbridge, James Currey, 225 p.

Kantel, Donald J. (2006) Laughter, Holy, in Burgess, S. M. (éd.) Encyclopedia of Pentecostal and Charismatic Christianity, London, Routledge, pp. 287-288.

Kaoues, Fatiha (2017) Migrantes au Liban. L'église évangélique comme mode d'intégration sociale, Hommes \& migrations, $n^{\circ} 1319$, pp. 41-48.

Kaoues, Fatiha (2014) Réfugiés et migrants dans les églises protestantes évangéliques libanaises : Recompositions identitaires et enjeux sociaux, L'Année du Maghreb, n 11, pp. 195-211.

Kergoat, Danièle (2009) Dynamique et consubstantialité des rapports sociaux, in Dorlin, E. (éd.) Sexe, race, classe : pour une épistémologie de la domination, Paris, Presses universitaires de France, $313 \mathrm{p}$.

Krause, Kristine (2011) Cosmopolitan charismatics? Transnational ways of belonging and cosmopolitan moments in the religious practice of New Mission Churches, Ethnic and Racial Studies, vol. 34, n³, pp. 419-435.

Kutlutürk, Bülent (2010) Zirve : Geliyorum diyen katliam! [Zirve : The massacre that was seen miles away !] in Çavdar, A. ; Yıldırım, A. B. (éds.) İstanbul, Uluslararası Hrant Dink Vakfı Yayınları, pp. 119-131.

Lalive d'Épinay, Christian (2007) Le combat avec l'ange. À propos d'une étude du mouvement pentecôtiste au Chili, Sociologies (La recherche en actes) [Disponible sur Internet].

Leiris, Michel (1996) Miroir de l' Afrique, Paris, Gallimard, 1476 p. (Quatro). 
Leman, Johan (2007) A "Lucan Effect" in the Commitment of Iranian Converts in Transit. The case of the Pentecostal Iranian Enclave in Istanbul, Revue des mondes musulmans et de la Méditerranée, $\mathrm{n}^{\circ}$ 119-120, pp. 101-114 [Disponible sur Internet].

Lenclud, Gérard (1996) Le grand partage ou la tentation ethnologique, in Althabe, G.; Fabre, D. ; Lenclud, G. (éds.) Vers une ethnologie du présent, Paris, Éditions de la Maison des Sciences de l'Homme, pp. 9-37.

Levitt, Peggy (2003) "You Know, Abraham Was Really the First Immigrant" : Religion and Transnational Migration, International Migration Review, vol. 37, n³, pp. 847-873.

Luca, Nathalie (2008a) Individus et pouvoirs face aux sectes, Paris, Armand Colin, $279 \mathrm{p}$.

Luca, Nathalie (2008b) Postface. Entre l'ethnie et la secte : les dérives de l'essentialisme, Archives de sciences sociales des religions, $\mathrm{n}^{\circ}$ 143, pp. 235-251 [Disponible sur Internet].

Lyman, Stanford ; Douglass, William (1973) Ethnicity : Strategies of Collective and Individual Impression Management, Social Research, vol. 40, n², pp. 344-365.

Magnani, José ; Cantor, Guilherme (2009) Etnografia como prática e experiência, Horizontes Antropológicos, vol. 15, n 32, pp. 129-156.

Mahieddin, Émir Chamil (2015) Faire le travail de Dieu. Une anthropologie morale du pentecôtisme en Suède contemporaine, Aix-Marseille, Université d'Aix-Marseille, 524 p.

Th. Doct. : Anthropologie : Université d'Aix-Marseille : 2015..

Marcillat, Audrey ; Miramond, Estelle ; Rupert, Nouri (2017) Introduction : l'intersectionnalité à l'épreuve du terrain, Les Cahiers du CEDREF, n² 21, pp. 7-15.

Mariano, Ricardo (2001) Análise sociológica do crescimento pentecostal no Brasil, São Paulo, Universidade de São Paulo, 285 p.

Martiniello, Marco ; Simon, Patrick (2005) Les enjeux de la catégorisation. Rapports de domination et luttes autour de la représentation dans les sociétés post-migratoires, Revue européenne des migrations internationales, vol. 21, $\mathrm{n}^{\circ} 2$, pp. 7-18.

Mary, André (2002) Le pentecôtisme brésilien en Terre africaine. L'universel abstrait du Royaume de Dieu, Cahiers d'études africaines, vol. 42, n 167, pp. 463-478.

Mary, André (2000) L'anthropologie au risque des religions mondiales, Anthropologie et Sociétés, vol. $24, \mathrm{n}^{\circ} 1$, pp. 117-135.

Maskens, Maïté (2013) Cheminer avec Dieu : pentecôtismes et migrations à Bruxelles, Bruxelles, Éd. de l'Université de Bruxelles, 217 p.

Mézié, Nadège (2010) “Wi, se kretyènn mwen ye” [Yes I am Christian]. Methodological Falsehood in Fieldwork, Fieldwork in Religion, vol. 5, n², pp. 180-192.

Millet-Mouity, Pamela (2017) Enquêter sur la sexualité des néo-pentecôtistes de la région parisienne. Retour sur l'expérience d'une socio-anthropologue parmi les « siens », Émulations, $\mathrm{n}^{\circ} 23$, pp. 13-26.

Morice, Alain (2005) Le cheval et l'alouette. Variations pour Althabe sur le thème de l'implication, Journal des anthropologues, $\mathrm{n}^{\circ}$ 102-103, pp. 503-29.

Mossière, Géraldine (2014) Réseaux pentecôtistes, activités d'évangélisation et émotions partagées parmi des Congolais établis à Montréal : "un cosmopolitisme de charisme" ? in Fer, Y. ; Malogne-Fer, G. (dir.) Le protestantisme évangélique à l'épreuve des cultures, Paris, L'Harmattan, pp. 107-128. 
Mossière, Géraldine (2007) Sharing in ritual effervescence : emotions and empathy in fieldwork, Anthropology Matters, vol. 9, $\mathrm{n}^{\circ}$ 1, [Disponible sur Internet].

Mottier, Damien (2014) Une ethnographie des pentecôtismes africains en France : le temps des prophètes, Louvain-la-Neuve, Academia ; l'Harmattan, 382 p.

Mottier, Damien ; Oro, Ari Pedro (2012) Entrepreneuriat charismatique et dynamiques transatlantiques de reconquête spirituelle (Amérique du Sud/Afrique/Europe), in Argyriadis, K. ; Capone, S. ; Torre, R. de la ; Mary, A. (dir.) Religions transnationales des Suds Afrique, Europe, Amériques, Louvain-la-Neuve, Academia ; l'Harmattan, pp. 193-212.

Muñoz, José Esteban (1999) Disidentifications : queers of color and the performance of politics, Minneapolis, University of Minnesota Press, 227 p.

Novaes, Regina (1985) Os escolhidos de Deus : pentecostais, trabalhadores e cidadania, Rio de Janeiro, Marco Zero, 158 p.

Olivier de Sardan, Jean-Pierre (2000) Le "je" méthodologique. Implication et explicitation dans l'enquête de terrain, Revue française de sociologie, vol. 41, n 3, pp. 417-445.

Olivier de Sardan, Jean-Pierre (1995) La politique du terrain. Sur la production des données en anthropologie, Enquête, Archives de la revue Enquête, n 1, pp. 71-109.

Olivier de Sardan, Jean-Pierre (1988) Jeu de la croyance et "je” ethnologique : exotisme religieux et ethno-égo-centrisme, Cahiers d'Études africaines, vol. 28, n 111, pp. 527-540.

Oro, Ari Pedro ; Stael, Carlos Alberto ; Rickli, João (2012) Introdução, in Oro, A. P. ; Stael, C. A. ; Rickli, J. (dir.) Transnacionalização religiosa : fluxos e redes, São Paulo, Terceiro Nome, pp. 7-15.

Ouattara, Fatoumata (2004) Une étrange familiarité. Les exigences de l'anthropologie « chez soi ", Cahiers d'études africaines, $\mathrm{n}^{\circ}$ 175, pp. 635-658.

Palomares, Élise ; Tersigni, Simona (2001) Les rapports de place dans l'enquête : les ressources du malentendu, Langage et société, vol. 97, n 3, pp. 5-26.

Papadopoulos, Dimitris ; Stephenson, Niamh ; Tsianos, Vassilis (2008) Escape routes : control and subversion in the twenty-first century, London, Ann Arbor, Pluto Press, $300 \mathrm{p}$.

Pfefferkorn, Roland (2011) Rapports de racisation, de classe, de sexe..., Migrations Société, $\mathrm{n}^{\circ}$ 133, pp. 193-208.

Picard, Julie (2013) Le Caire des migrants africains chrétiens : impasse migratoire et citadinités religieuses, Paris, EHESS, $466 \mathrm{p}$.

Price-Mars, Jean (1973) Ainsi parla l'oncle, Montréal, Leméac, 316 p.

Renahy, Nicolas (2010) Classes populaires et capital d'autochtonie, Regards sociologiques, $\mathrm{n}^{\circ} 40$, pp. 9-26.

Richter, Philip (1997) The Toronto Blessing : Charismatic Evangelical Global Warming, in Hunt, S. ; Hamilton, M. ; Tony, W. (dir.) Charismatic Christianity. Sociological Perspectives, Basingstoke, Macmillan, pp. 97-119.

Scrinzi, Francesca (2008) Quelques notions pour penser l'articulation des rapports sociaux de "race", de classe et de sexe, Les Cahiers du CEDREF, n 16, pp. 81-99.

Simon, Patrick (2000) Les jeunes issus de l'immigration se cachent pour vieillir : représentations sociales et catégories de l'action publique, Ville-École-Intégration, Enjeux, n 121, pp. 23-38. 
Soothill, Jane E. (2007) Gender, social change, and spiritual power : charismatic christianity in Ghana, Leiden, Brill, $261 \mathrm{p}$.

Suter, Brigitte (2012) Tales of Transit. Sub-Saharan African Migrants' Experiences in Istanbul, Malmö, Linköping University ; Malmö University, 307 p.

Tassin, Etienne (2008) Condition migrante et citoyenneté cosmopolitique : des manières d'être soi et d'être au monde, Dissensus, pp. 2-19.

Trawalé, Damien (2017) Être interpellé en tant que gay noir dans la France contemporaine. Tensions subjectives et hiérarchisations identificatoires, Les Cahiers du CEDREF, $\mathrm{n}^{\circ}$ 21, pp. 103-129.

Turner, Victor (1990) Le phénomène rituel : structure et contre-structure, Paris, Presses universitaires de France, 206 p.

Velho, Gilberto (2015) Métropole, cosmopolitisme et médiation, Ateliers d'anthropologie, $\mathrm{n}^{\circ} 41$, [Disponible sur Internet].

Wyart, Aude ; Fait, Nicolas (2013) Le hasard peut-il bien faire les choses ? La sérendipité à travers deux récits croisés de terrain, Sociologies, (Dossier) [Disponible sur Internet].

Wyk, Ilana van (2013) Beyond ethical imperatives in South African anthropology : Morally repugnant and unlikeable subjects, Anthropology Southern Africa, vol. 36, $\mathrm{n}^{\circ}$ 1\&2, pp. 68-79.

Yıldırım, Uğur (2005) Dünü, Bugünü, İçyüzü ve Perde Arkasıyla Türkiye'de Misyonerlik, İstanbul, Otopsi Yayınları, $430 \mathrm{p}$.

Young, Pauline Vislick (2006) Les pèlerins de Russian-Town : le combat d'une société religieuse primitive pour survivre dans un environnement urbain, Paris, L'Harmattan, $287 \mathrm{p}$.

\section{NOTES}

1. Sur le paradigme associant pentecôtisme et déracinement, voir Aupiais-L'homme, 2017.

2. L'intérêt pour la décharge émotionnelle dans les pratiques religieuses des populations déplacées se retrouve dans des littératures nord-américaines plus anciennes sur les religiosités de descendants d'esclaves africains (Price-Mars 1973 [1928]), ou d'immigrés forcés européens (Young, 2006 [1932]).

3. Églises qui se développent de part et d'autre de frontières étatico-nationales. Dans la suite du texte "Église " désigne une communauté de foi ou une organisation; et "église ", un lieu de culte.

4. Nos corpus d'analyse contiennent une centaine d'heures d'observation et trois entretiens semi-directifs enregistrés à Paris (2011-2013) dans le cadre d'un mémoire de master, une centaine d'heures d'observation et six entretiens semi-directifs enregistrés à Istanbul (2013-2016) dans le cadre de ce même mémoire et d'une thèse de doctorat en cours.

5. Enquêtes ethnographiques et enquêtes policières emploient des procédures semblables telles que la triangulation (Sardan 1995).

6. Sur le lien entre migration et désidentification, voir Papadopoulos et al. 2008. Toute migration ne se fait évidemment pas hors de ces espaces d'assignation, mais l'ethnographie des désidentification prend moins pour objet la circulation contrainte que l'installation dans des espaces a priori non affectés par la structure de l'économie : ici le Brésil, pour des pentecôtistes racialisés d'Europe de l'Ouest ; la Turquie, pour des évangéliques appauvris des pays du Sud.

7. Outre la réflexion pionnière de Erving Goffman (1975) sur l'usage de "désidentificateurs » (attitudes, gestes, paroles) par les stigmatisés pour déjouer une dépréciative; la seule théorisation de la désidentification en sciences sociales remonte à notre connaissance au travail 
de José Esteban Muñoz (1999), qui prend pour objet la contestation de l'idéologie hétéronormative dans les performances queer. Nous proposons donc une nouvelle acception du terme.

8. Précisons comme l'illustre magistralement Philippe Gonzalez (2014) dans le sillage de Jeanne Favret-Saada, que l'ethnographe rechignant à participer activement au travail religieux peut de toute façon être profondément affecté, et son incrédulité mise en jeu.

9. Un rapport social est une « relation antagonique entre deux groupes sociaux, établie autour d'un enjeu " (Kergoat, 2009), une tension macrosociale au sens où elle préexiste à la mise en relation entre les acteurs concrets, et préside leur catégorisation. La sociologie matérialiste des rapports sociaux a particulièrement analysé les rapports de sexe, de « race » et de classe, parfois d'âge et d'orientation sexuelle (Scrinzi, 2008) et leurs imbrications, ou intersections. Pour des débats actuels en langue française, voir Marcillat et al. 2017.

10. Nous reprenons à notre compte la définition minimale d'autochtone comme «individu ou population originaire du pays ou de la région qu'il habite» (Simon et al., 1993), en l'occurrence des personnes primo-socialisées en France hexagonale et plus spécifiquement en région parisienne.

11. L'ethnographie pionnière de Hermione Harris prend place à Londres entre 1969 et 1974.

12. Nous entendons par là une intégration des schèmes culturels spécifiques de l'Universelle à l'univers de sens des populations européennes.

13. Ce que Xavier Dunezat (2015) qualifie de catégorisation ex post.

14. Les membres de la FJF sont répartis en "tribus », dont les noms reprennent ceux des douze tribus d'Israël selon la Bible : Ephraïm, Juda, Nephtali etc.

15. Dans son enquête sur les gays noirs, Damien Trawalé a bien montré combien race et ethnicité - la première étant souvent retenue comme construction naturaliste, et la seconde comme construction culturaliste, de l'altérité - tendaient à s'amalgamer dans la France contemporaine (Trawalé, 2017).

16. Sur la distinction entre contextes structurel « historique » et conjoncturel « immédiat », voir Palomares \& Tersigni, 2001.

17. Ce mot est d'ailleurs une catégorie officielle de fidèles à l'Universelle, distincte des « personnes du monde » sans aucun lien avec l'Église (Gomes, 2011).

18. Le "témoignage» est un dispositif narratif pentecôtiste actant, par le récit d'une transformation générale et/ou d'un événement miraculeux face à l'ensemble des fidèles, le salut du témoignant.

19. Le récit indigène retient comme événement fondateur la " bénédiction de Toronto ", en 1994 (Richter, 1997).

20. La Turquie applique une limitation géographique au traité de Genève et ne reconnaît officiellement le statut de réfugié qu'au ressortissants de pays d'« Europe ». L'obtention du statut de réfugié par les demandeurs d'asile afghans, iraniens ou syriens implique donc normalement leur réinstallation dans un pays tiers par le Haut-Commissariat aux Réfugiés, raison pour laquelle l'étude de l'asile est souvent confondue en Turquie avec l'étude du transit migratoire.

21. Fatiha Kaoues (2014) appelle aussi à relativiser, au Liban, les ressources sociales réellement mises à dispositions des migrants par les églises.

22. Nous qualifions d' "évangélistes » les membres de l'église dans le cadre de leur pratique d'évangélisation.

23. Le nombre de personnes (en centaines de milliers) "sauvées" par des missionnaires de l'organisation Cascade à l'échelon international fut longtemps affiché sur son site internet, sous forme d'un compteur en perpétuel mouvement. En avril 2019, le site déclare avoir sauvé plus de 17750000 âmes.

24. Condamnant fermement la migration des Africains francophones vers l'Europe de l'ouest, et raillant les pasteurs qui bénissent leurs fidèles avant leur passage - «Ouvrir les portes? Et 
pourquoi? Vous allez où? Qu'allez-vous faire là-bas?»- le pasteur-assistant, originaire du Nigeria critiquait en particulier l'influence des « émotions » dans la prise de décision et l'acte de migrer (mars 2015). Ceci illustre une double-tendance de la Cascade à promouvoir la mobilité internationale des uns (demandeurs d'asile chrétiens originaires du Moyen-Orient) en proscrivant celle des autres (femmes et hommes pentecôtistes, originaires d'Afrique de l'Ouest).

25. Installé à Istanbul depuis plus de dix ans et jouissant d'une forte notabilité dans plusieurs communautés de foi, cet «entrepreneur charismatique» (Mottier \& Oro, 2012) et d'autres responsables religieux font partie, au même titre que les gérants de restaurants rencontrés par Nicolas Fait à Istanbul, « des personnes installées depuis un temps que l'on pourrait juger bien trop long pour que le qualificatif de transit vienne qualifier sans équivoque la situation dans laquelle ils se trouvaient " (Wyart \& Fait, 2013).

26. Voir à ce sujet les rapports sur les violations des droits humains publiés en turc et en anglais par l'Association des Églises Protestantes [Protestan Kiliseler Derneği] de Turquie.

27. La responsable camerounaise du groupe d'évangélisation en langue française nous lançait: «Faut pas croire qu'ici c'est que pour les Africains » (mars 2014).

28. Au début de l'enquête, les quelques «cours » de langue française que nous dispensions aux enfants de la bourgeoisie stambouliote coûtaient moins en temps et rapportaient plus d'argent que les gardes d'enfants effectués par nos interlocutrices originaires des pays du Sud, sans compter notre patrimoine et notre bourse Erasmus. L'obtention d'un contrat doctoral hisserait plus tard nos revenus à un niveau presque inatteignable pour ces dernières.

29. Sur le double sens de l'ancrage comme «mouillage» donnant accès à des ressources relationnelles, et « amarrage » défini par la dépendance momentanée au milieu, voir Debarbieux, 2014.

30. Sur la distinction entre pratique ethnographique "programmée et continue ", et expérience ethnographique « imprévue et discontinue », voir Magnani, 2009.

\section{RÉSUMÉS}

Cet article discute les articulations théoriques entre pentecôtisme et migrations en comparant la participation d'un ethnographe à la marge du travail religieux de deux Églises, et les identifications dont il fait l'objet au regard de ses positions relatives dans les rapports sociaux. À partir de ces enquêtes chez soi mais non parmi les siens, puis autre mais parmi d'autres autres, nous considérons les aspects désidentificatoires de la conversion, de la migration, et de l'ethnographie.

\section{INDEX}

Index géographique : France, Turquie

Mots-clés : communauté religieuse, pratique religieuse, relations sociales, ethnographie 


\section{AUTEUR}

\section{ARMAND AUPIAIS}

Doctorant en socio-anthropologie à l'URMIS (Université Paris Diderot); Membre de l'axe

« Migrations \& Mobilités » (IFEA, Istanbul)

armand.al@protonmail.com 